\title{
The Effect of Sodium-Glucose Cotransporter 2 Inhibitor Ipragliflozin on Insulin Resistance, Hepatic Insulin Clearance, Beta-Cell Function in the Japanese Patients with type 2 Diabetes
}

\section{Tsuyoshi Okura ( $\nabla$ ohkura@med.tottori-u.ac.jp )}

Tottori University Faculty of Medicine https:// orcid.org/0000-0003-3713-6617

Yohei Fujioka

Tottori University: Tottori Daigaku

Risa Nakamura

Tottori University: Tottori Daigaku

Sonoko Kitao

Tottori University: Tottori Daigaku

Yuichi Ito

Tottori University: Tottori Daigaku

Mari Anno

Tottori University: Tottori Daigaku

Kazuhisa Matsumoto

Tottori University: Tottori Daigaku

Kyoko Shoji

Tottori University: Tottori Daigaku

Kazuhiko Matsuzawa

Tottori University: Tottori Daigaku

Shoichiro Izawa

Tottori University: Tottori Daigaku

Hiroko Okura

Tottori University: Tottori Daigaku

Etsuko Ueta

Tottori University: Tottori Daigaku

Masahiko Kato

Tottori University: Tottori Daigaku

Takeshi Imamura

Tottori University: Tottori Daigaku

Shin-ichi Taniguchi

Tottori University: Tottori Daigaku 


\section{Kazuhiro Yamamoto}

Tottori University: Tottori Daigaku

\section{Research}

Keywords: Sodium-glucose cotransporter 2 inhibitor, Insulin Clearance, Type 2 Diabetes, Insulin Clamp

Posted Date: September 14th, 2021

DOI: https://doi.org/10.21203/rs.3.rs-882630/v1

License: (c) (1) This work is licensed under a Creative Commons Attribution 4.0 International License. Read Full License 
1 The effect of sodium-glucose cotransporter 2 inhibitor ipragliflozin on

2 insulin resistance, hepatic insulin clearance, beta-cell function in the

3 Japanese patients with type 2 diabetes

4

5 Tsuyoshi Okura ${ }^{1 *}$, Yohei Fujioka ${ }^{1}$, Risa Nakamura ${ }^{1}$, Sonoko Kitao ${ }^{1}$, Yuichi Ito ${ }^{1}$, Mari

6 Anno $^{1}$, Kazuhisa Matsumoto ${ }^{1}$, Kyoko Shoji ${ }^{1}$, Kazuhiko Matsuzawa ${ }^{1}$, Shoichiro Izawa ${ }^{1}$,

7 Hiroko Okura ${ }^{1}$, Etsuko Ueta ${ }^{2}$, Masahiko Kato ${ }^{2}$, Takeshi Imamura ${ }^{3}$, Shin-ichi

8 Taniguchi $^{4}$, Kazuhiro Yamamoto ${ }^{1}$

9

$10 \quad{ }^{1}$ Division of Cardiovascular Medicine, Endocrinology and Metabolism, Tottori

11 University Faculty of Medicine

$12{ }^{2}$ School of Health Science, Tottori University Faculty of Medicine

$13 \quad{ }^{3}$ Division of Molecular Pharmacology, Tottori University Faculty of Medicine

$14 \quad{ }^{4}$ Department of Regional Medicine, Tottori University Faculty of Medicine

15

16 Short title: The effect of SGLT2-i

17

18

19 "Corresponding author, and name and address of the person to whom reprint requests:

20 Tsuyoshi Okura, MD, Ph.D., Assistant Professor, Division of Cardiovascular Medicine,

21 Endocrinology and Metabolism, Tottori University Faculty of Medicine, 36-1 Nishi-

22 Cho, Yonago, Tottori 683-8504, Japan

23 E-mail: ohkura@tottori-u.ac.jp

24 Telephone: $+81-859-386517$ 
25 Fax: +81-859-386519

26 ORCID ID: 0000-0003-3713-6617

27

28 Word count: abstract 244 main text 2238

29 Number of figures 1

$30 \quad$ Number of tables 2

31 


\section{Abstract}

33 Introduction: Sodium-glucose cotransporter 2 inhibitor (SGLT2i) is a medication for

34 type 2 diabetes mellitus (T2DM). Some reports showed SGLT2i improved insulin

35 resistance, however, the effect on insulin resistance is not well established. Hepatic

36 insulin clearance (HIC) is new pathophysiology of T2DM. The effect of SGLT2i on

37 hepatic insulin clearance and insulin resistance is not well known. We investigated the

38 effect of SGLT2i on insulin resistance, insulin secretion, incretins, body composition,

39 and hepatic insulin clearance.

40 Materials and Methods: We conducted a meal tolerance test (MTT), and the

41 hyperinsulinemic-euglycemic clamp in $9 \mathrm{~T} 2 \mathrm{DM}$ patients. $50 \mathrm{mg} /$ day ipragliflozin was

42 admitted, MTT and clamp were performed after 4 months. We calculated the

43 postprandial C-peptide AUC to insulin AUC ratio as the HIC. We also measured GLP-

44 1, GIP, and glucagon levels during MTT.

45 Results: Body weight, HbA1c, and body composition were not significantly changed

46 after 4 months of treatment. Postprandial glucose, fasting, and postprandial insulin were

47 significantly decreased. The insulin resistance of the glucose clamp was not changed,

48 but HOMA-IR and insulin sensitivity index (ISI) were significantly improved. Incretins

49 and glucagon were not changed. Hepatic insulin clearance was significantly increased,

50 but whole-body insulin clearance was not changed. Fib 4 index and fatty liver index

51 were significantly reduced. HOMA-beta and insulinogenic index was not changed but

52 the C-peptide index was significantly increased. 
53 Conclusions: Although patients' number was small, these results suggest that SGLT2i

54 treatment decreased hepatic insulin resistance, increased hepatic insulin clearance, and

55 decreased hyperinsulinemia, it might protect beta-cell function.

\section{Keywords}

58 Sodium-glucose cotransporter 2 inhibitor; Insulin Clearance; Type 2 Diabetes; Insulin 59 Clamp

60

\section{Abbreviations}

62 CPI, C-peptide index; eGFR, estimated glomerular filtration rate; FLI, fatty liver index;

63 GDR, glucose disposal rate; GIP, glucose-dependent insulinotropic polypeptide; GLP-1, 64 glucagon-like peptide-1; HIC, hepatic insulin clearance; HOMA-beta, homeostatic model 65 assessment beta-cell function; HOMA-IR, homeostasis model assessment for insulin 66 resistance, SGLT2i, Sodium-glucose cotransporter 2 inhibitor

67

68

69 


\section{Introduction}

71 The main pathophysiology of type 2 diabetes mellitus (T2DM) is decreased insulin

72 sensitivity and decreased insulin secretion ability (1). Sodium-glucose cotransporter 73 inhibitor (SGLT2i) is an anti-diabetic medicine, which increases glucosuria, induces a 74 glucose-lowering effect, and reduces body weight (2). Some studies reported SGLT2i to 75 improve insulin resistance. Dapagliflozin improved muscle insulin resistance evaluated 76 by the euglycemic hyperinsulinemic clamp technique (3). However, this study and other 77 studies indicated SGLT2i increases endogenous glucose production and glucagon 78 secretion. One study performed dual-tracer glucose administrated mixed meal, and 79 showed SGLT2i increased insulin sensitivity and the secretion of glucagon-like peptide801 (GLP-1), but increased endogenous glucose production, and increased glucagon section

81 (4). Another study performed a glucose clamp method and oral glucose tolerance test, 82 SGLT2i improved insulin sensitivity (5). These results suggested that SGLT2i improve 83 muscle insulin sensitivity, but increase endogenous glucose production by glucagon 84 secretion.

85 Hepatic insulin clearance (HIC) is a novel and important pathophysiology of hyper86 insulinemia, metabolic syndrome, and T2DM, hepatic insulin clearance is decreased in 87 these metabolic conditions (6). One study reported that SGLT2i increased insulin 88 clearance after the meal tolerance test (7). However, we previously reported that poorly 89 controlled T2DM patients showed increased hepatic insulin clearance (8).

90 According to these results, SGLT2i affects insulin resistance, incretins, hepatic insulin 91 clearance, however, these effects were evaluated by different studies, therefore the total 
110 We performed this study according to the principles of the Declaration of Helsinki. The 111 Ethics Committee of the Tottori University Faculty of Medicine approved this study 112 (approval number 2439). The clinical trial number was UMIN000014521.

mechanisms are not well known in the real world. We evaluated the effect of SGLT2i on insulin resistance measured by the glucose clamp method, and we also evaluated the effect on hepatic insulin clearance and beta-cell function. We also measured incretins as GLP-1, and glucose-dependent insulinotropic polypeptide (GIP), and glucagon levels in the meal tolerance test. Here, we show that SGLT2i improves hepatic insulin resistance and increases hepatic insulin clearance, improves liver function, and it might protect betacell function.

\section{Materials and Methods}

\section{Subjects}

Nine type 2 diabetes patients enrolled in the study at Tottori University Hospital from 2014 to 2017. We diagnosed type 2 diabetes by the World Health Organization (WHO) criteria (9). We excluded patients with malignancy, hepatitis B and C, liver cirrhosis, pancreatitis, renal dysfunction, or those who taking diabetogenic medications such as corticosteroids from the study. All patients were of Japanese ethnicity. This study had a single-arm study design. $50 \mathrm{mg} /$ day ipragliflozin was admitted after meal tolerance test (MTT) and glucose clamp test, and MTT and clamp were performed after 4 months. Patients were lectured to drink water to prevent dehydration. 
114 Trial registration: UMIN, UMIN000014521. Registered 9 July 2014 - Retrospectively

115 registered, https://upload.umin.ac.jp/cgi-open-

$116 \mathrm{bin} / \mathrm{ctr} / \mathrm{ctr}$.cgi?function=brows\&action=brows\&type=summary\&language $=\mathrm{J} \& \mathrm{recptno}=$

117 R000016186

118 The Patient and Public Involvement

119 The first patient was involved on May 7, 2014. The patient was recruited from the out-

120 patient of Tottori University hospital. The research questions were developed in the Ethics

121 Committee and informed to all of the patients using a procedure that was approved by the

122 Ethics Committee. This study was a single-arm and intervention study. We chose the

123 outcome HOMA-IR and Glucose disposal rate as a marker of insulin resistance. All

124 patients were recruited from out-patients of Tottori University hospital. We obtained

125 informed consent from all of the patients using a procedure that was approved by the

126 Ethics Committee.

\section{Meal tolerance test (MTT)}

129 The patients visited our hospital after overnight fasting. The patients took a test meal

130 developed by the Japan Diabetes Society (JDS) (460 kcal, carbohydrate 50\%, protein 15\%,

131 fat 35\%, and salt 1.6 g) (10). Plasma glucose, serum insulin, and C-peptide were measured

132 at $0,30,60$, and 120 minutes after taking the test meal. We used the glucose oxidase

133 method for glucose, and chemiluminescent immunoassays for insulin and C-peptide

134 (human insulin, and C-peptide chemiluminescent immunoassay kits; Kyowa Medix, 
135 Japan). HbA1c was measured by high-performance liquid chromatography. HbA1c was

136 converted from the percentage values to the International Federation of Clinical

137 Chemistry values ( $\mathrm{mmol} / \mathrm{mol}), \mathrm{HbA} 1 \mathrm{c}$ converter from the National Institutes of Diabetes

138 and Digestive and Kidney Diseases was used (11).

139 Insulin resistance was assessed by using insulin resistance indexes calculated as follows:

140 Homeostatic model assessment insulin resistance $($ HOMA-IR $)=\{$ fasting plasma glucose

$141(\mathrm{mmol} / \mathrm{L})\} \times\{$ fasting plasma insulin $(\mathrm{pmol} / \mathrm{L})\} / 135(12)$.

142 Insulin sensitivity index $($ ISI $)=10,000 /$ square $[\{$ fasting plasma glucose $(\mathrm{mmol} / \mathrm{L}) \times$

143 fasting plasma insulin $(\mathrm{pmol} / \mathrm{L})\} \times\{$ mean glucose $\times$ mean insulin during the MTT $\}]$ 144 (13).

145 Insulin secretion was assessed by using insulin secretion indexes as follows:

146 Homeostasis model assessment of beta cell function (HOMA-beta) $=[20 \times\{$ fasting

147 plasma insulin $(\mathrm{pmol} / \mathrm{L})\}] /[\{$ fasting plasma glucose $(\mathrm{mmol} / \mathrm{L})\}-3.5](12)$.

148 Insulinogenic index $=[\{$ plasma insulin $(\mathrm{pmol} / \mathrm{L})$ at $30 \mathrm{~min}\}-\{$ plasma insulin $(\mathrm{pmol} / \mathrm{L})$

149 at $0 \mathrm{~min}\}] /[\{$ plasma glucose $(\mathrm{mmol} / \mathrm{L})$ at $30 \mathrm{~min}\}-\{$ plasma glucose $(\mathrm{mmol} / \mathrm{L})$ at 0

$150 \min \}]$ 8).

151 C-peptide index $(\mathrm{CPI})(14)=\{$ fasting C-peptide $(\mathrm{mmol} / \mathrm{L})\} /\{$ fasting plasma glucose $152(\mathrm{mmol} / \mathrm{L}) \times 100\}$

153 We calculated Hepatic insulin clearance (HIC) as follows: 
$154 \mathrm{HIC}=\{$ area under the curve $($ AUC $)$ C-peptide 0-120 min $\} /\{$ AUC insulin 0-120 min $)\}$

155 (15). The AUC was calculated using the trapezoidal method.

157 GLP-1, GIP, and glucagon assays

158 We obtained the serum samples by using a blood collection tube containing DPP-4

159 inhibitor (BD ${ }^{\mathrm{TM}}$ P800, BD Japan, Japan). We performed ethanol and solid-phase

160 extractions for assay (16). We measured intact GLP-1 levels by an ELISA kit (GLP-1

161 (active) ELISA, Merck Millipore, Germany). We also measured the active GIP by an

162 ELISA kit (Human GIP, Active form Assay Kit, IBL, Japan). We next measured

163 Glucagon levels by an ELISA kit (Glucagon ELISA kit, Mercodia, Sweden).

\section{Hyperinsulinemic euglycemic clamp}

166 The glucose clamp method was performed as we previously reported $(8,17)$. We

167 performed the hyperinsulinemic-euglycemic clamp method to evaluate insulin sensitivity

168 by an artificial endocrine pancreas (STG 55, Nikkiso, Japan). We performed the protocol

169 as a priming constant insulin infusion $\left(100 \mathrm{mU} / \mathrm{m}^{2} / \mathrm{min}\right)$, and we maintained the plasma

170 glucose level at $5.2 \mathrm{mmol} / \mathrm{L}(95 \mathrm{mg} / \mathrm{dL})$ during the clamp. The plasma insulin level of

171 steady-state was achieved as $1200 \mathrm{pmol} / \mathrm{L}$ in T2DM patients based on previous studies

172 (18). We measured the glucose infusion rate (GIR) of steady-state from 90-120 minutes,

173 and we defined the mean GIR during this period as the glucose disposal rate (GDR). GDR

174 is generally used as mainly reflecting peripheral insulin sensitivity. We next calculated 
insulin concentration in the clamp, which is peripheral insulin sensitivity adjusted by final

177 insulin concentration (19). The whole-body insulin clearance was calculated as the insulin

178 infusion rate divided by the steady-state insulin concentration (19).

\section{$179 \quad$ Fib 4 index}

180 Liver fibrosis level was estimated by Fib 4 index $=$ age $([\mathrm{yr}]$ AST $[\mathrm{U} / \mathrm{L}]) /\left(\left(\mathrm{PLT}\left[10^{9} / \mathrm{L}\right]\right)\right.$

$\left.181(\mathrm{ALT}[\mathrm{U} / \mathrm{L}])^{1 / 2}\right)(20)$

182 Fatty liver index (FLI)

183 Fatty liver level was estimated by

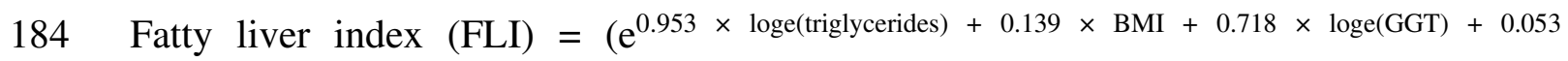

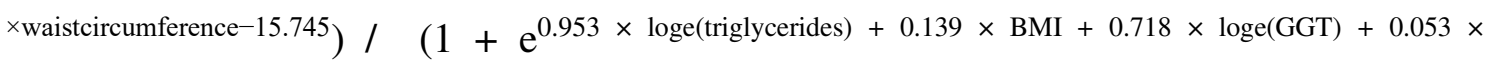
waistcircumference-15.745) $\times 100(21)$.

\section{Body composition}

188 Body composition was analyzed by Inbody 770 (Inbody Japan, Japan). Body water,

189 Percent body fat, Body fat mass, Lean body mass, Skeletal muscle mass, Trunk fat mass,

190 and Bone mineral mass were measured.

\section{Statistical analysis}

192 Data are expressed as the mean \pm standard deviation. We assessed differences in the mean

193 value of clinical parameters between before and after SGLT2i treatment by using the

194 Mann-Whitney $U$ test. We determined correlations analysis of non-parametric clinical

195 variables by using Spearman's correlation analysis. Values of $P<0.05$ were considered 
196 to be significant. PRISM8 software (GraphPad Software, San Diego, USA) was used for 197 all analyses.

198 We conducted a power analysis to compare the pretreatment and posttreatment HOMA199 IR as a primary endpoint using an EZR calculator 23. The mean HOMA-IR in the 200 pretreatment was $4.86 \pm 1.14$, and the mean HOMA-IR in the posttreatment was $3.48 \pm$ 201 1.53. The difference between the mean HOMA-IR in the pre and post-treatment was 1.38 $202 \pm 0.39$. We conducted a power analysis of the statistical test that was used to compare the 203 pre and post-treatment HOMA-IR. Assuming that the difference in the means was 1.38 204 and the SD was 0.39 , alpha $=0.05,1$-beta $=0.8, R=0.57$, the estimated sample size was 9 205 patients.

206 
209 The mean age of subjects was $54.1 \pm 9.1$ years old, and 2 males, 7 females. Duration of

210 Diabetes was $5.0 \pm 4.1$ years. Participant characteristics are shown in Table 1. Seven

211 patients were only diet therapy, 2 patients take DPP4 inhibitor and alpha-glucosidase

212 inhibitor, and 1 patient also take metformin. Table 1 shows 4 months' effects of SGLT2i.

213 Bodyweight, HbA1c, and body composition were not significantly changed after 4

214 months of treatment. AST, fib 4 index, and fatty liver index were significantly decreased.

215 Postprandial glucose, fasting and postprandial insulin were decreased. Figure 1 shows

216 MTT results. Glucose 120 minutes after MTT was significantly decreased. Fasting and

21760,120 minutes insulin levels were significantly decreased. C-peptide, GLP-1, GIP, and 218 glucagon levels were not changed. The insulin resistance of the glucose clamp was not 219 changed, but HOMA-IR and insulin sensitivity index were significantly improved.

220 Incretins were not changed. Hepatic insulin clearance was significantly increased. The 221 whole-body insulin clearance was not changed. HOMA-beta and insulinogenic index was 222 not changed but the C-peptide index was significantly increased.

223 Table 2 shows the correlation between delta C-peptide index and delta clinical parameters 224 from pretreatment to after treatment. The hepatic insulin clearance was significantly 225 correlated with CPI. Creatinine and eGFR were also correlated with CPI. 


\section{Discussion}

232 Our study indicated that SGLT2i ipragliflozin improved HOMA-IR, liver function, and

233 C-peptide index, but did not change significantly the muscle insulin resistance evaluated

234 by glucose clamp method, and body weight, body composition. SGLT2i increased hepatic 235 insulin clearance, but not whole-body insulin clearance. A recent study indicated that

236 SGLT2i improved insulin resistance evaluated by glucose clamp (22). Dapagliflozin

237 reduces liver fat but did not affect tissue insulin sensitivity of the liver, muscle, and

238 adipocyte (23). Recent another study also reported that SGLT2i increased hepatic insulin 239 clearance (24). SGLT2i increases glucagon section from alpha cells (25). We did not see 240 the significant glucagon inhibition by SGLT2i, but it may depend on the number of small 241 patients number, and 2 patients were already taking DPP4i.

242 According to these results, SGLT2i decreased postprandial glucose levels and insulin

243 levels. HOMA-IR mainly reflects liver insulin resistance, and the glucose clamp method 244 mainly reflects muscle insulin resistance (26). Although the insulin resistance of muscle 245 evaluated by the glucose clamp method was not improved, liver insulin resistance 246 evaluated by HOMA-IR was considered as improved. Hyperinsulinemia was decreased 247 after SGLT2i treatment, this insulin lowering effect was induced by increased hepatic 248 insulin clearance. These effects induced improved liver function. Finally, the C-peptide 249 index was increased, which might mean SGLT2i has the effect of beta-cell protection. 250 Although further basic research of animals and cellular are needed for understanding 251 molecular mechanisms, our results contribute to understanding the effects of SGLT2i. A 252 recent American study showed similar results that SGLT2i canagliflozin improved liver 253 insulin sensitivity and beta-cell function, and increased hepatic insulin clearance (27). 
254 However, the pathophysiology of diabetes between American, European and Asian

255 people are very different, Asian shows low insulin secretion ability and insulin resistance 256 with mild obesity $(28,29)$. All patients in our study are Japanese, therefore, our results 257 contribute to the daily clinical work in the Asian population.

258 Our study has some limitations, the major limitation is the very small patients' number of 259 nine. However, the glucose clamp method and meal tolerance test including incretins are 260 very complicated and expensive studies. We need a larger study. We evaluated insulin 261 resistance by the glucose clamp method. The previous report evaluated GDR by 262 subtracting the urinary glucose excretion (3). We measured the urinary glucose extraction 263 in only 2 patients, therefore we cannot evaluate the urinary glucose excretion, we must 264 consider this point. We did not evaluate hepatic insulin resistance by tritium-glucose, 265 because of the difficulty of using tritium-glucose in Japan. We could evaluate hepatic 266 insulin resistance by using only HOMA-IR, we need furthermore study. We used the 267 MTT to avoid hyperglycemia by using OGTTs in patients with severe diabetes. However, 268 the previous study used the HIC obtained from MTT, therefore we think our study was acceptable (7). Although our main purpose was to evaluate hepatic insulin clearance,

270 whole-body and peripheral insulin clearance are also important as previously reported 271 (30). Unfortunately, the steady-state C-peptide levels in the glucose clamp were not 272 measured in this study, we need to investigate the peripheral insulin clearance evaluated 273 during the glucose clamp test. The recent article stated that the post-load CPR / IRI molar 274 ratio did not accurately evaluate hepatic insulin clearance (31). We must be careful to 275 evaluate our results. We evaluated beta-cell function by C-peptide index. C-peptide index 276 is affected by renal function shown as correlation analysis. We excluded renal disorder 
$277 \mathrm{Cr}>1.3 \mathrm{mg} / \mathrm{ml}$ according to a past report (31), and eGFR did not show significant change

278 therefore we consider our data are reliable. Despite these limitations, our study performed

279 glucose clamp and meal tolerance test and incretins measurement in the Japanese patients

280 with T2DM in the real-world setting, we consider that our study might contribute to our

281 daily clinical work, especially in the Asian population.

282 In conclusion, SGLT2i decreased postprandial glucose and insulin levels, increased

283 hepatic insulin clearance, and improved liver function and liver insulin resistance, it might

284 protect beta-cell function.

286 Acknowledgments

287 We thank Ms. Satomi Endo for her excellent technical assistance.

288

289 Contributors: All authors contributed significantly. TO participated in the design of the 290 study and performed the statistical analysis. YF, RN, SK, YI, MA, KM, SK, KM, SI,

291 HO, and EU collected the data. MK, TI, ST, and KY conceived the study, participated

292 in its design and coordination, and helped to draft the manuscript. All authors read and

293 approved the final manuscript. 
296 This study was approved by the Ethics Committee of the Faculty of Medicine, Tottori

297 University (approval number 2439). Clinical trial number was UMIN000014521

298

299 Funding: This study was funded by Astellas Pharma Inc. No grant number.

300 The company was not involved in study design, patient selection, data

301 aggregation/analysis, interpretation of results, or preparation of the manuscript.

302

303 Competing interests: None declared.

304

305 Data sharing statement: No additional data available.

306

307

308 
References

310 1. Kahn SE, Cooper ME, Del Prato S. Pathophysiology and treatment of type 2 diabetes:

311 perspectives on the past, present, and future. Lancet 2014;383:1068-1083

312 2. Ohkura T. Ipragliflozin: A novel sodium-glucose cotransporter 2 inhibitor developed

313 in Japan. World J Diabetes. 2015;6:136-44.

314 3. Merovci A, Solis-Herrera C, Daniele G, Eldor R, Fiorentino TV, Tripathy D, Xiong J,

315 Perez Z, Norton L, Abdul-Ghani MA, DeFronzo RA. Dapagliflozin improves muscle

316 insulin sensitivity but enhances endogenous glucose production. $\mathrm{J}$ Clin Invest.

$317 \quad 2014 ; 124: 509-14$.

318 4. Ferrannini E, Muscelli E, Frascerra S, Baldi S, Mari A, Heise T, Broedl UC, Woerle

319 HJ. Metabolic response to sodium-glucose cotransporter 2 inhibition in type 2 diabetic 320 patients. J Clin Invest. 2014;124:499-508.

321 5. Mudaliar S, Henry RR, Boden G, Smith S, Chalamandaris AG, Duchesne D, Iqbal N,

322 List J. Changes in insulin sensitivity and insulin secretion with the sodium glucose 323 cotransporter 2 inhibitor dapagliflozin. Diabetes Technol Ther. 2014;16:137-44.

324 6. Pivovarova O, Bernigau W, Bobbert T, Isken F, Möhlig M, Spranger J, Weickert MO,

325 Osterhoff M, Pfeiffer AF, Rudovich N. Hepatic insulin clearance is closely related to 326 metabolic syndrome components. Diabetes Care 2013;36:3779-85

327 7. Ekholm E, Hansen L, Johnsson E, Iqbal N, Carlsson B, Chen H, Hirshberg B. 328 COMBINED TREATMENT WITH SAXAGLIPTIN PLUS DAPAGLIFLOZIN 

Taniguchi SI, Yamamoto K. Hepatic insulin clearance is increased in patients with high HbA1c type 2 diabetes: a preliminary report. BMJ Open Diabetes Res Care. 2020;8:e01149.

9. Alberti KG, Zimmet PZ: Definition, diagnosis and classification of diabetes mellitus and its complications. Part 1: diagnosis and classification of diabetes mellitus provisional report of a WHO consultation. Diabet Med 1998;15:539-553

10. Yoshino G, Tominaga M, Hirano T, Shiba T, Kashiwagi A, Tanaka A, Tada N, meal A: A pilot model for the international standard of test meal for an assessment of both postprandial hyperglycemia and hyperlipidemia. J Jpn Diabetes Soc 2006;49:361371 (In Japanese)

11. National Institutes of Diabetes and Digestive and Kidney Diseases, the HbA1c converter, [article online]. 1999. Available from: http://www.ngsp.org/convert1.asp. 12. Matthews DR, Hosker JP, Rudenski AS, Naylor BA, Treacher DF, Turner RC.

347 Homeostasis model assessment: insulin resistance and beta-cell function from fasting 348 plasma glucose and insulin concentrations in man. Diabetologia 1985;28:412-419

349 13. Matsuda M, DeFronzo RA. Insulin sensitivity indices obtained from oral glucose 350 tolerance testing: comparison with the euglycemic insulin clamp. Diabetes Care. 1999, 22: $1462-1470$. 
353 A, Fujita H, Hara K, Kadowaki T, Tobe K: Genetic risk score constructed using 14 354 susceptibility alleles for type 2 diabetes is associated with the early onset of diabetes and 355 may predict the future requirement of insulin injections among Japanese individuals. 356 Diabetes Care. 2012, 35: 1763-1770.

357 15. Pivovarova O, Bernigau W, Bobbert T, Isken F, Möhlig M, Spranger J, Weickert MO, 358 Osterhoff M, Pfeiffer AF, Rudovich N. Hepatic insulin clearance is closely related to 359 metabolic syndrome components. Diabetes Care. 2013;36:3779-85.

360 16. Yabe D, Eto T, Shiramoto M, Irie S, Murotani K, Seino Y, Kuwata H, Kurose T, 361 Seino S, Ahrén B, Seino Y. Effects of DPP-4 inhibitor linagliptin and GLP-1 receptor 362 agonist liraglutide on physiological response to hypoglycaemia in Japanese subjects with type 2 diabetes: A randomized, open-label, 2-arm parallel comparative, exploratory trial.

364 Diabetes Obes Metab 2017;19:442-447

365 17. Ohkura T, Shiochi H, Fujioka Y, Sumi K, Yamamoto N, Matsuzawa K, Izawa S, 366 Kinoshita H, Ohkura H, Kato M, Taniguchi SI, Yamamoto K. 20/(fasting C-peptide x 367 fasting plasma glucose) is a simple and effective index of insulin resistance in patients with type 2 diabetes mellitus: a preliminary report. Cardiovasc Diabetol 2013;12:21 18. Tamura Y, Tanaka Y, Sato F, Choi JB, Watada H, Niwa M, Kinoshita J, Ooka A, 370 Kumashiro N, Igarashi Y, Kyogoku S, Maehara T, Kawasumi M, Hirose T, Kawamori R.

371 Effects of diet and exercise on muscle and liver intracellular lipid contents and insulin sensitivity in type 2 diabetic patients. J Clin Endocrinol Metab 2005;90:3191-3196 19. DeFronzo RA, Tobin JD, Andres R. Glucose clamp technique: a method for

374 quantifying insulin secretion and resistance. Am J Physiol 1979;237:214-223 
375

376

377

378

379

380

381

382

383

384

385

386

387

388

389

390

391

392

393

394

395

396

20. Sterling RK, Lissen E, Clumeck N, Sola R, Correa MC, Montaner J, S Sulkowski M, Torriani FJ, Dieterich DT, Thomas DL, Messinger D, Nelson M; APRICOT Clinical Investigators. Development of a simple noninvasive index to predict significant fibrosis in patients with HIV/HCV coinfection. Hepatology. 2006;43:1317-25.

21. Bedogni G, Bellentani S, Miglioli L, Masutti F, Passalacqua M, Castiglione A, Tiribelli C. The Fatty Liver Index: a simple and accurate predictor of hepatic steatosis in the general population. BMC Gastroenterol. 2006;6:33.

22. So A, Sakaguchi K, Okada Y, Morita Y, Yamada T, Miura H, Otowa-Suematsu N, Nakamura T, Komada H, Hirota Y, Tamori Y, Ogawa W. Relation between HOMA-IR and insulin sensitivity index determined by hyperinsulinemic-euglycemic clamp analysis during treatment with a sodium-glucose cotransporter 2 inhibitor. Endocr J. 2020;67:501507.

23. Latva-Rasku A, Honka MJ, Kullberg J, Mononen N, Lehtimäki T, Saltevo J, Kirjavainen AK, Saunavaara V, Iozzo P, Johansson L, Oscarsson J, Hannukainen JC, Nuutila P. The SGLT2 Inhibitor Dapagliflozin Reduces Liver Fat but Does Not Affect Tissue Insulin Sensitivity: A Randomized, Double-Blind, Placebo-Controlled Study With 8-Week Treatment in Type 2 Diabetes Patients. Diabetes Care. 2019;42:931-937.

24. Matsubayashi Y, Yoshida A, Suganami H, Osawa T, Furukawa K, Suzuki H, Fujihara K, Tanaka S, Kaku K, Sone H. Association of increased hepatic insulin clearance and change in serum triglycerides or beta-hydroxybutyrate concentration via the sodium/glucose-cotransporter 2 inhibitor tofogliflozin. Diabetes Obes Metab. 2020;22:947-956. 

D, Sasaki T, Takeuchi K, Kakizaki S, Yamada M, Kitamura T. SGLT1 in pancreatic $\alpha$ 399 cells regulates glucagon secretion in mice, possibly explaining the distinct effects of $400 \quad$ SGLT2 inhibitors on plasma glucagon levels. Mol Metab. 2019;19:1-12.

26. Muniyappa R, Lee S, Chen H, Quon MJ. Current approaches for assessing insulin 402 sensitivity and resistance in vivo: advantages, limitations, and appropriate usage. Am $\mathrm{J}$ 403 Physiol Endocrinol Metab. 2008;294:E15-26. Kalavalapalli S, Pettus J, Ciaraldi TP, Mudaliar S, Henry RR. Effect of canagliflozin treatment on hepatic triglyceride content and glucose metabolism in patients with type 2 diabetes. Diabetes Obes Metab. 2019;21:812-821.

28. Hsu WC, Araneta MR, Kanaya AM, Chiang JL, Fujimoto W, BMI cut points to 409 identify at-risk Asian Americans for type 2 diabetes screening. Diabetes Care. 2015; 38: $410 \quad 150-8$

411 29. Okura T, Nakamura R, Fujioka Y, Kawamoto-Kitao S, Ito Y, Matsumoto K, Shoji K,

412 Sumi K, Matsuzawa K, Izawa S, Ueta E, Kato M, Imamura T, Taniguchi SI, Yamamoto $413 \mathrm{~K}$. Body mass index $\geq 23$ is a risk factor for insulin resistance and diabetes in Japanese 414 people: A brief report. PLoS One. 2018;13:e0201052.

415 30. Elahi D, Nagulesparan M, Hershcopf RJ, Muller DC, Tobin JD, Blix PM, Rubenstein $416 \mathrm{AH}$, Unger RH, Andres R. Feedback inhibition of insulin secretion by insulin: relation to 417 the hyperinsulinemia of obesity. N Engl J Med. 1982;306:1196-202. 
418 31. Funakoshi S, Fujimoto S, Hamasaki A, et al. Analysis of factors influencing 419 pancreatic beta-cell function in Japanese patients with type 2 diabetes: association with 420 body mass index and duration of diabetic exposure. Diabetes Res Clin Pract 2008;82:3534218.

422 32. Piccinini F, Bergman RN. The Measurement of Insulin Clearance. Diabetes Care. $423 \quad 2020 ; 43: 2296-2302$. 


\section{Figure Legends}

426 Figure 1. Figure 1 shows the meal tolerance test (MTT) results. Figure 1(a) shows

427 glucose levels after the MTT, (b) insulin, (c) C-peptide, (d) GLP-1, (e) GIP, and (f)

428 glucagon. White circles represent the data from pre-treatment, and black squares

429 indicate post treatment. *; $p<0.05$

430 
Table 1. Comparison between before and after SGLT2 treatment

\begin{tabular}{|c|c|c|c|}
\hline Parameters & before & after & $P$ value \\
\hline Body weight (kg) & $71.5 \pm 9.1$ & $70.2 \pm 9.8$ & 0.12 \\
\hline $\operatorname{BMI}\left(\mathrm{kg} / \mathrm{m}^{2}\right)$ & $28.2 \pm 4.0$ & $27.7 \pm 4.0$ & 0.11 \\
\hline Waist circumstance $(\mathrm{cm})$ & $96.9 \pm 9.2$ & $96.5 \pm 9.6$ & 0.39 \\
\hline eGFR & $86.2 \pm 23.9$ & $85.6 \pm 24.3$ & 0.84 \\
\hline AST (U/L) & $28.1 \pm 7.7$ & $24.2 \pm 8.7$ & $<0.05$ \\
\hline ALT (U/L) & $32.3 \pm 14.6$ & $28.5 \pm 13.5$ & 0.12 \\
\hline Gamma GTP (U/L) & $42.5 \pm 47.3$ & $37.3 \pm 41.0$ & 0.11 \\
\hline Fib 4 index & $0.92 \pm 0.30$ & $0.77 \pm 0.32$ & $<0.05$ \\
\hline Fatty liver index & $59.8 \pm 23.2$ & $51.3 \pm 27.9$ & $<0.05$ \\
\hline HbA1c $(\%)$ & $7.4 \pm 1.1$ & $6.8 \pm 0.5$ & 0.09 \\
\hline $\mathrm{HbA1c}(\mathrm{mmol} / \mathrm{mol})$ & $57.4 \pm 12.0$ & $50.8 \pm 5.8$ & \\
\hline \multicolumn{4}{|l|}{ Insulin secretion } \\
\hline Fasting Plasma Glucose (mmol/L) & $7.28 \pm 1.35$ & $6.83 \pm 1.26$ & 0.41 \\
\hline Postprandial Plasma Glucose 2h (mmol/L) & $9.94 \pm 2.50$ & $8.25 \pm 2.30$ & $<0.05$ \\
\hline Fasting Plasma insulin $(\mathrm{mmol} / \mathrm{L})$ & $90.4 \pm 20.9$ & $69.9 \pm 32.6$ & $<0.01$ \\
\hline Postprandial Plasma insulin $2 \mathrm{~h}(\mathrm{mmol} / \mathrm{L})$ & $285.3 \pm 122.9$ & $175.1 \pm 111.2$ & $<0.05$ \\
\hline Glucose AUC (0-120 min) & $362.4 \pm 62.8$ & $331.1 \pm 61.5$ & 0.17 \\
\hline Insulin AUC (0-120 min) & $568.1 \pm 250.9$ & $370.9 \pm 293.9$ & 0.08 \\
\hline CPR AUC (0-120 min) & $3.28 \pm 1.33$ & $2.90 \pm 0.81$ & 0.35 \\
\hline Hepatic insulin clearance & $6.03 \pm 1.46$ & $9.96 \pm 4.26$ & $<0.05$ \\
\hline HOMA-beta (\%) & $87.4 \pm 32.7$ & $78.5 \pm 46.3$ & 0.30 \\
\hline Insulinogenic Index & $0.62 \pm 0.25$ & $0.63 \pm 0.55$ & 0.98 \\
\hline CPI & $1.92 \pm 0.79$ & $2.25 \pm 0.66$ & $<0.05$ \\
\hline \multicolumn{4}{|l|}{ Insulin resistance } \\
\hline HOMA-IR & $4.86 \pm 1.14$ & $3.48 \pm 1.53$ & $<0.05$ \\
\hline Insulin sensitivity index & $2.70 \pm 0.81$ & $4.67 \pm 2.56$ & $<0.05$ \\
\hline GDR (mg/kg/min) & $5.52 \pm 1.66$ & $5.68 \pm 2.63$ & 0.77 \\
\hline $\mathrm{M} / \mathrm{I}$ & $4.97 \pm 1.60$ & $6.07 \pm 4.38$ & 0.65 \\
\hline Whole-body insulin clearance & $1.37 \pm 0.28$ & $1.36 \pm 0.39$ & 0.95 \\
\hline \multicolumn{4}{|l|}{ Incretins } \\
\hline GLP-1 AUC & $14.8 \pm 9.1$ & $15.8 \pm 11.2$ & 0.41 \\
\hline GIP AUC & $126.1 \pm 75.6$ & $106.3 \pm 27.5$ & 0.30 \\
\hline Glucagon AUC & $13.3 \pm 5.1$ & $12.7 \pm 5.4$ & 0.44 \\
\hline \multicolumn{4}{|l|}{ Body composition } \\
\hline Body water (L) & $34.3 \pm 4.2$ & $33.7 \pm 4.9$ & 0.09 \\
\hline Percent body fat (\%) & $34.2 \pm 7.6$ & $34.5 \pm 7.7$ & 0.49 \\
\hline Body fat mass (kg) & $24.7 \pm 7.8$ & $24.6 \pm 7.9$ & 0.72 \\
\hline Lean body mass (kg) & $43.9 \pm 5.5$ & $43.2 \pm 6.4$ & 0.1 \\
\hline Skeletal muscle mass (kg) & $25.6 \pm 3.5$ & $25.1 \pm 4.1$ & 0.1 \\
\hline Trunk fat mass (kg) & $14.6 \pm 5.5$ & $15.2 \pm 5.5$ & 0.53 \\
\hline Bone mineral mass $(\mathrm{kg})$ & $2.6 \pm 0.3$ & $2.5 \pm 0.4$ & 0.34 \\
\hline
\end{tabular}

Data are presented as the mean \pm standard deviation. 
433 The comparison of parameters between before and after treatment was performed using

434 the Mann-Whitney $U$ test. The chi-squared test was used for categorical comparisons of 435 the sex data.

436

437 AUC, area under the curve; CPI, C-peptide index; GDR, glucose disposal rate; GIP, 438 glucose-dependent insulinotropic polypeptide; GLP-1, glucagon-like peptide-1; HbA1c, 439 glycated hemoglobin; HOMA-beta, homeostatic model assessment beta cell function; 440 HOMA-IR, homeostasis model assessment for insulin resistance; N.S., not significant 441 
Table 2. Correlation coefficients between the $\Delta \mathrm{CPI}$ and clinical parameters

\begin{tabular}{lcc}
\hline Parameters & $\mathrm{r}$ & $\mathrm{p}$ \\
\hline$\Delta$ Fasting plasma glucose & -0.334 & $\mathrm{NS}$ \\
$\Delta$ Postprandial plasma glucose 120min & -0.366 & $\mathrm{NS}$ \\
$\Delta$ Fasting insulin & -0.133 & $\mathrm{NS}$ \\
$\Delta$ Postprandial insulin 120min & 0.158 & $\mathrm{NS}$ \\
$\Delta$ Matsuda Index & 0.533 & 0.07 \\
$\Delta$ HOMA-IR & -0.466 & 0.106 \\
$\Delta$ Insulinogenic Index & 0.266 & $\mathrm{NS}$ \\
$\Delta$ HIC=AUC CPR/INS & 0.833 & $<0.005$ \\
$\Delta$ TG(0) & -0.400 & $\mathrm{NS}$ \\
$\Delta$ AST & 0.252 & $\mathrm{NS}$ \\
$\Delta$ ALT & -0.218 & $\mathrm{NS}$ \\
$\Delta \gamma$ GTP & -0.292 & $\mathrm{NS}$ \\
$\Delta$ Fatty liver index & 0.274 & $\mathrm{NS}$ \\
$\Delta$ Fib 4 index & 0.144 & $\mathrm{NS}$ \\
$\Delta$ HDL & 0.033 & $\mathrm{NS}$ \\
$\Delta$ LDL & 0.133 & $\mathrm{NS}$ \\
$\Delta$ HbA1c & -0.235 & $\mathrm{NS}$ \\
$\Delta$ eGFR & 0.733 & $<0.05$ \\
$\Delta$ GDR & 0.567 & 0.06 \\
$\Delta$ M/I & 0.250 & $\mathrm{NS}$ \\
$\Delta$ Whole body insulin clearance & 0.410 & $\mathrm{NS}$ \\
$\Delta$ BMI & -0.117 & $\mathrm{NS}$ \\
$\Delta$ Waist circumstance & -0.516 & $\mathrm{NS}$ \\
$\Delta$ Total body fat & 0.183 \\
$\Delta$ GLP-1 AUC120 & -0.100 & $\mathrm{NS}$ \\
$\Delta$ GIP AUC120 & -0.100 & \\
$\Delta$ Glucagon AUC120 & -0.100 & \\
\hline
\end{tabular}

444

445 Correlation coefficients were determined using Spearman's product-moment correlation 446 coefficient test.

447 AUC, area under the curve; GDR, glucose disposal rate; GIP, glucose-dependent 448 insulinotropic polypeptide; GLP-1, glucagon-like peptide-1; HbA1c, glycated 449 hemoglobin; HOMA-beta, homeostatic model assessment beta-cell function; HOMA-IR, 450 homeostasis model assessment for insulin resistance; N.S., not significant 
451 Figure 1
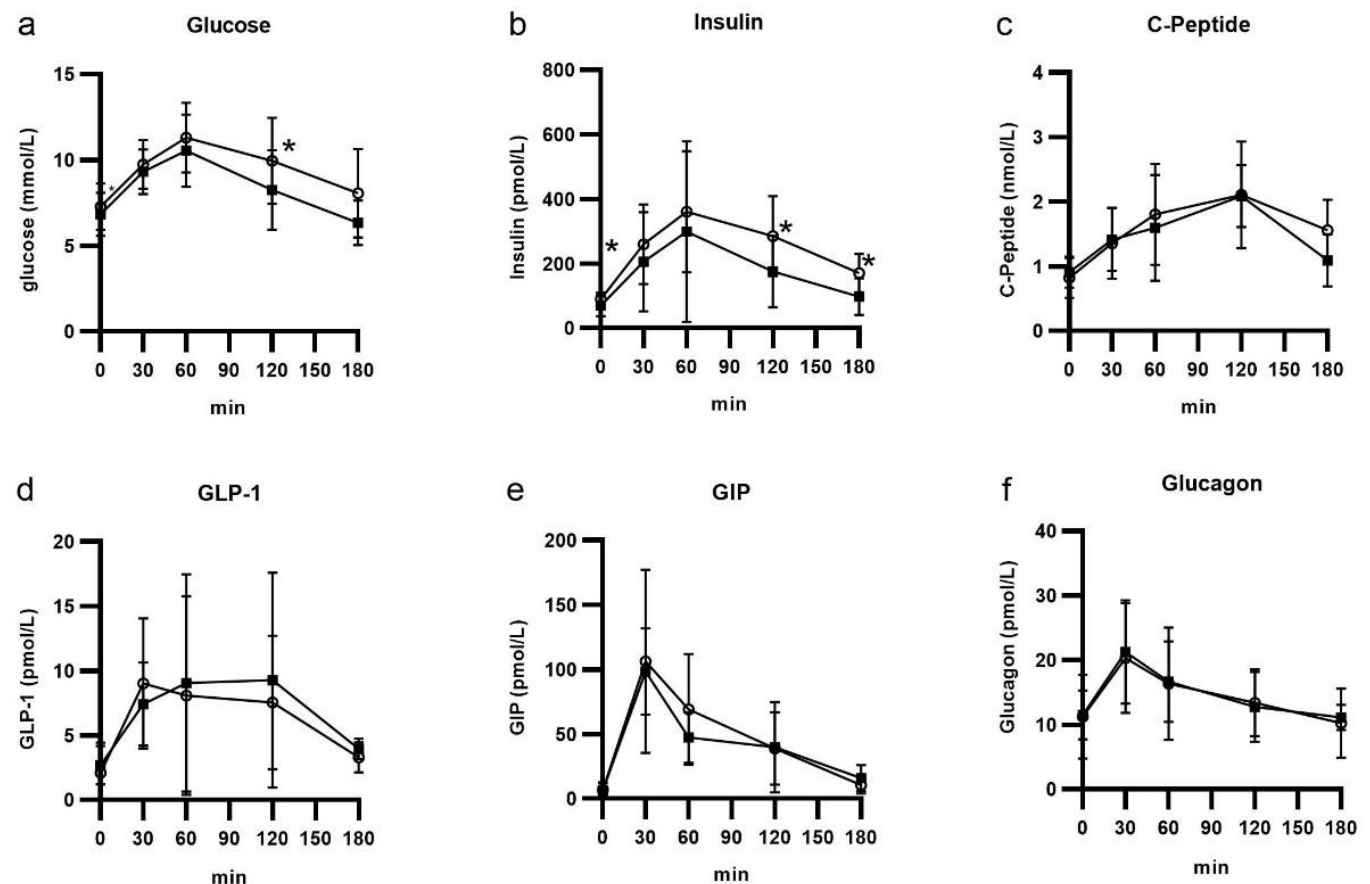